\title{
Quantum Coherence and Its Signatures in Extended Quantum Systems
}

\author{
Rajesh Dutta ${ }^{1}$ and Biman Bagchi ${ }^{1, *}$ \\ ${ }^{1}$ SSCU, Indian Institute of Science, Bangalore 560012, India. \\ Corresponding Author
}

*bbagchi@iisc.ac.in

\section{Supporting Information (SI)}

\section{STOCHASTIC DIFFUSION OPERATOR FOR POISSON AND GAUSSIAN PROCESS AND PARAMETERS FOR THE SYSTEM HAMILTONIAN}

For Poisson bath the stochastic diffusion operator $\Gamma$ can be expressed as $2 \times 2$ matrix as follows,

$$
\Gamma=\left(\begin{array}{cc}
-\frac{b}{2} & \frac{b}{2} \\
\frac{b}{2} & -\frac{b}{2}
\end{array}\right)
$$

with the eigenvalues 0 and $-b$.

However, for Gaussian-Markov process the stochastic diffusion operator is a Fokker-Planck operator and provided as follows

$$
\Gamma_{\mathrm{V}}=b \frac{\partial}{\partial \mathrm{V}}\left(\mathrm{V}+\frac{\partial}{\partial \mathrm{V}}\right)
$$


Table S1: Site energies and electronic coupling energies of first three subunits of FMO complex are given in $\mathrm{cm}^{-1}$.

\begin{tabular}{|l|l|l|l|}
\hline $\begin{array}{l}\text { Site } \\
\text { number }\end{array}$ & $\mathbf{1}$ & $\mathbf{2}$ & $\mathbf{3}$ \\
\hline $\mathbf{1}$ & 12410 & -87.7 & 5.5 \\
\hline $\mathbf{2}$ & -87.7 & 12530 & 30.8 \\
\hline $\mathbf{3}$ & 5.5 & 30.8 & 12210 \\
\hline
\end{tabular}

For the dimer system we consider first two sites from the above table.

\section{COUPLED EQUATION OF MOTION}

The coupled equation of motion for same bath case can be given as follows

$$
\begin{aligned}
\frac{d \sigma_{m}}{d t}= & -\frac{i}{\hbar} H_{S}^{x} \sigma_{m}-\frac{i}{\hbar} V_{d} \sum_{m^{\prime}=0}^{1}\left(\delta_{m+1, m^{\prime}}+\delta_{m-1, m^{\prime}}\right) \times\left(\sum_{k}|k\rangle\langle k|\right)^{x} \sigma_{m^{\prime}} \\
& -\frac{i}{\hbar} V_{o d} \sum_{m^{\prime}=0}^{1}\left(\delta_{m+1, m^{\prime}}+\delta_{m-1, m^{\prime}}\right) \times\left(\sum_{\substack{k, l \\
k \neq l}}|k\rangle\langle l|\right)^{x} \sigma_{m^{\prime}}-m b \sigma_{m}
\end{aligned}
$$

Here, subscript denotes the eigen states of bath. The interaction between system-bath occurs via the $\mathrm{V}_{\mathrm{d}}$ and $\mathrm{V}_{\text {od. }}$. The Kronecker delta correspond to the transition from equilibrium bath states to excited bath states and vice versa.

The Eq. (S3) can be obtained after expansion of reduced density matrix in terms of eigen state of the bath followed by the eigen value of the bath states. 
The diagonal fluctuation $V_{d}(t)$ and off-diagonal fluctuation $V_{o d}(t)$ of the time dependent Hamiltonian (Eq. 5 and 6) have the following matrix elements in the eigen-functions of the stochastic diffusion operator $\Gamma$ as follows

$\left\langle b_{i}\left|V_{a}(t)\right| b_{j}\right\rangle=V_{a}$, where $i \neq j$ and $\left\langle b_{i}\left|V_{a}(t)\right| b_{i}\right\rangle=0$.

$\left|b_{i}\right\rangle$ is the eigen state of the stochastic diffusion operator.

\section{SOLUTION OF COUPLED EQUATION OF MOTION}

One can write the coupled equation of motion as follows

$\dot{\boldsymbol{X}}(t)=\boldsymbol{A} \boldsymbol{X}(t)$

where, $\boldsymbol{X}(t)$ is a $8 \times 1$ matrix for correlated bath model whereas it is a $32 \times 1$ for uncorrelated bath

model. $\dot{\boldsymbol{X}}(t)$ is the first derivatives, and $\boldsymbol{A}$ is an $8 \times 8$ matrix for correlated bath model and $32 \times 32$

matrix for uncorrelated bath.

Laplace transform of (S4) will provide the following equation

$s \tilde{\boldsymbol{X}}(s)-\boldsymbol{X}(0)=\boldsymbol{A} \tilde{\boldsymbol{X}}(s)$

where, $\mathbf{X}(0)$ is the initial condition i.e. initially excitation is placed on site 1.

From (A5) one can get all the functions in Laplace domains as follows

$$
\begin{aligned}
& (s \boldsymbol{I}-\boldsymbol{A}) \tilde{\boldsymbol{X}}(s)=\boldsymbol{X}(0) \\
& \tilde{\boldsymbol{X}}(s)=(s \boldsymbol{I}-\boldsymbol{A})^{-1} \boldsymbol{X}(0)
\end{aligned}
$$


where, $\boldsymbol{I}$ is an identity matrix. Now, using $s \rightarrow 0$ limit followed by inverse Laplace transform of the resulting function, we obtain Eq. (15) to Eq. (19).

\section{CONSTANTS OF EQUILIBRIUM POPULATIONS AND COHERENCES FOR THE UNCORRELATED BATH MODEL}

We provide all the constants presents in the equation equilibrium and population and coherence for uncorrelated bath model as follows

$$
\begin{aligned}
& k_{1}=V_{d} b_{d}\left(64 a^{4} b_{d}^{6} c e^{3}-40 a^{2} b_{d}^{8} c e^{3}+32 a^{2} b_{d}^{6} c^{3} e^{3}+8 a^{2} b_{d}^{6} c e^{5}\right) \\
& k_{2}=512 a^{6} b_{d}^{7} c^{2}-640 a^{4} b_{d}^{9} c^{2}+128 a^{2} b_{d}^{11} c^{2}+512 a^{4} b_{d}^{7} c^{4}-128 a^{2} b_{d}^{9} c^{4} \\
& +32 a^{2} b_{d}^{7} c^{2} e^{4}+128 a^{2} b_{d}^{7} c^{4} e^{2}-160 a^{2} b_{d}^{9} c^{2} e^{2}+256 a^{4} b_{d}^{7} c^{2} e^{2} \\
& k_{3}=V_{d}^{2} b_{d}^{2}\left(128 a^{6} b_{d}^{5} c^{2}-160 a^{4} b_{d}^{7} c^{2}-32 a^{2} b_{d}^{9} c^{2}-256 a^{4} b_{d}^{5} c^{4}-32 a^{2} b_{d}^{7} c^{4}\right) \\
& +V_{d}^{2} b_{d}^{2}\left(32 a^{4} b_{d}^{5} c^{2} e^{2}+16 a^{2} b_{d}^{7} c^{2} e^{2}-64 a^{2} b_{d}^{5} c^{4} e^{2}+16 a^{2} b_{d}^{5} c^{2} e^{4}\right) \\
& k_{4}=V_{d}^{4} b_{d}^{4}\left(32 a^{4} b_{d}^{3} c^{4}+16 a^{2} b_{d}^{5} c^{4}\right) \\
& k_{5}=V_{d} b_{d}\left(\begin{array}{c}
-256 a^{7} b_{d}^{6} c+320 a^{5} b_{d}^{8} c-64 a^{3} b_{d}^{10} c-256 a^{5} b_{d}^{6} c^{3}+64 a^{3} b_{d}^{8} c^{3} \\
+80 a^{3} b_{d}^{8} c e^{2}-128 a^{5} b_{d}^{6} c e^{2}-64 a^{3} b_{d}^{6} c^{3} e^{2}-16 a^{3} b_{d}^{6} c e^{4}
\end{array}\right) \\
& k_{6}=V_{d}^{3} b_{d}^{3}\left(64 a^{5} b_{d}^{4} c^{3}+32 a^{3} b_{d}^{6} c^{3}+16 a^{3} b_{d}^{4} c^{3} e^{2}\right) \\
& k_{7}=V_{d}^{3} b_{d}^{3}\left(\begin{array}{c}
-64 a^{7} b_{d}^{4} c+80 a^{5} b_{d}^{6} c-16 a^{3} b_{d}^{8} c-64 a^{5} b_{d}^{4} c^{3}+16 a^{3} b_{d}^{6} c^{3}+32 a^{5} b_{d}^{5} c e \\
-8 a^{3} b_{d}^{7} c e-16 a^{5} b_{d}^{4} c e^{2}+16 a^{3} b_{d}^{6} c e^{2}-16 a^{3} b_{d}^{4} c^{3} e^{2}+8 a^{3} b_{d}^{5} c e^{3}
\end{array}\right) \\
& k_{8}=V_{d}^{5} b_{d}^{5}\left(16 a^{5} b_{d}^{2} c^{3}+8 a^{3} b_{d}^{4} c^{3}\right) \\
& k_{9}=V_{d}^{2} b_{d}^{2}\left(\begin{array}{c}
128 a^{7} b_{d}^{5} c-160 a^{5} b_{d}^{7} c+32 a^{3} b_{d}^{9} c+128 a^{5} b_{d}^{5} c^{3}-32 a^{3} b_{d}^{7} c^{3} \\
+64 a^{5} b_{d}^{5} c e^{2}-40 a^{3} b_{d}^{7} c e^{2}+32 a^{3} b_{d}^{5} c^{3} e^{2}
\end{array}\right)
\end{aligned}
$$


$k_{10}=V_{d}^{4} b_{d}^{4}\left(-32 a^{5} b_{d}^{3} c^{3}-16 a^{3} b_{d}^{5} c^{3}+8 a^{3} b_{d}^{4} c^{3} e\right)$

and $\quad a=\frac{i J}{\hbar} ; c=\frac{i V_{d}}{\hbar} ; e=\frac{i\left(E_{1}-E_{2}\right)}{\hbar}$

Here, all the constants show the coupling and competition between off-diagonal coupling J, fluctuation strength $V_{d}$ and rate of fluctuation $b_{d}$ in establishing equilibrium population distribution.

\section{RELATION BETWEEN COHERENCE IN SITE BASIS AND EXCITONIC POPULATION}

The site basis and excitionic basis have the following relation

$\left\langle i\left|\sigma^{\text {site }}\right| j\right\rangle=\sum_{k, l}\left\langle i \mid e_{k}\right\rangle\left\langle e_{k}\left|\sigma^{e x c}\right| e_{l}\right\rangle\left\langle e_{l} \mid j\right\rangle$

where, $\mathrm{i}$ and $\mathrm{j}$ are the site basis and $\mathrm{e}_{\mathrm{k}}$ and $\mathrm{e}_{1}$ are the excitionic basis. $i, j, k, l \in\{1,2,3, \ldots \ldots . . . n\}$. In the long time limit the off-diagonal element in the excitionic basis disappears to establish the equilibrium distribution with respect to the diagonal elements. Hence, in the long time limit one can write Eq. (S17) as follows

$\left\langle i\left|\sigma^{\text {site }}\right| j\right\rangle=\sum_{k}\left\langle i \mid e_{k}\right\rangle\left\langle e_{k}\left|\sigma^{e x c}\right| e_{k}\right\rangle\left\langle e_{k} \mid j\right\rangle$

For $\mathrm{n}$ chromophoric system, coherence in site basis can be written as

$$
\left\langle 1\left|\sigma^{\text {site }}\right| n\right\rangle=\sum_{k=1}^{n}\left\langle 1 \mid e_{k}\right\rangle\left\langle e_{k}\left|\sigma^{e x c}\right| e_{k}\right\rangle\left\langle e_{k} \mid n\right\rangle
$$

Similarly there will be $\mathrm{n}-1$ equations i.e. total $\mathrm{n}$ equations. From the above equation it is clear that all the off diagonal elements in the site basis are connected with the diagonal elements in the excitionic basis via $\mathrm{n}$ coupled algebraic equations. 